\section{Utilização de adoçantes dietéticos entre adultos em Pelotas, Rio Grande do Sul, Brasil: um estudo de base populacional}

\author{
Use of diet sweeteners by adults in Pelotas, \\ Rio Grande do Sul State, Brazil: \\ a population-based study
}

\author{
1 Programa de Pós-graduação \\ em Epidemiologia, \\ Universidade Federal de \\ Pelotas, Pelotas, Brasil. \\ 2 Faculdade de \\ Nutrição,Universidade \\ Federal de Pelotas, Pelotas, \\ Brasil. \\ Correspondência \\ R. V. Zanini \\ Programa de Pós-graduação \\ em Epidemiologia, \\ Universidade Federal de \\ Pelotas. \\ Rua Marechal Deodoro 1160, \\ Pelotas, RS 96020-220, Brasil. \\ robe.nutri@gmail.com
}

\begin{abstract}
This population-based study evaluated the use of diet sweeteners by adults (> 20 years) in Pelotas, Rio Grande do Sul State, Brazil. Data were collected from January to July $2010(n=2,732)$. Besides specific questions on diet sweeteners, demographic, socioeconomic, and health characteristics were recorded. Heterogeneity and linear trend chi-square tests were used for the statistical analysis. Prevalence of sweetener use was 19\% (95\%CI: 1.1-20.9), and was 3.7 times higher in elderly individuals as compared to 20-29-year-olds. Economic level and nutritional status were significantly associated with sweetener use. Nearly $98 \%$ of the sample used liquid sweeteners; the most frequently consumed (89.2\%) were those containing saccharin or sodium cyclamate. Average intake was 10 drops of liquid sweetener or 1.5 sachets of powdered sweetener. Use of diet sweeteners was higher among women and the elderly.
\end{abstract}

Dietetic Sweeteners; Sweetening Agents; Prevalence
Roberta de Vargas Zanini 1

Cora Luiza Araújo 1,2

Jeovany Martínez-Mesa 1

\section{Introdução}

Até a década de 1980, no Brasil, os produtos dietéticos eram regulamentados como drogas, sendo comercializados em farmácias e consumidos apenas por quem necessitasse controlar a ingestão de sacarose, como portadores de diabetes mellitus ou de outras doenças ${ }^{1}$. Entretanto, a partir de 1988, os produtos à base de edulcorantes tiveram seu uso estendido para a população em geral ${ }^{2}$.

De acordo com a atual legislação brasileira, adoçantes dietéticos são "produtos formulados para utilização em dietas com restrição de sacarose, frutose e glicose, para atender às necessidades de pessoas sujeitas à restrição desses carboidratos" 3 . De forma geral, os adoçantes fazem parte de um grupo alimentar específico, denominado pelo Ministério da Saúde como "alimentos para fins especiais”, os quais são destinados a atender às necessidades de pessoas em condições metabólicas e fisiológicas específicas ${ }^{4}$. No entanto, os adoçantes dietéticos são produtos que estão atualmente disponíveis para os consumidores que desejam desfrutar do sabor doce sem aumentar seu consumo energético, pois, além de serem úteis para o controle glicêmico em pacientes diabéticos, podem ser uma ferramenta auxiliar na dietoterapia de outras morbidades, como a obesidade 5,6 .

Os adoçantes dietéticos são constituídos de edulcorantes não calóricos (naturais ou artifi- 
ciais), que conferem o sabor doce sem a adição de calorias. Nas últimas décadas, o consumo desse tipo de edulcorante tem aumentado em vários países 7 , e produtos dietéticos (especialmente refrigerantes diet e adoçantes dietéticos) têm sido as principais fontes de ingestão de edulcorantes não calóricos 1. O aumento global das doenças crônicas não transmissíveis, especialmente obesidade e diabetes mellitus, pode ser uma das justificativas para o consumo cada vez maior desse tipo de produto 8 .

No Brasil, a regulamentação do uso de edulcorantes é de responsabilidade do Ministério da Saúde, por meio da Agência Nacional de Vigilância Sanitária (ANVISA), com base em normas internacionais sobre o uso de aditivos em alimentos. Os tipos de edulcorantes não calóricos atualmente permitidos para comercialização no Brasil são: sacarina sódica, ciclamato de sódio, aspartame, acessulfame de potássio, sucralose, esteviosídeo, neotame e taumatina 9 .

Alguns estudos sobre o consumo de adoçantes têm sido realizados em populações específicas, geralmente abordando os efeitos do consumo de edulcorantes a longo prazo e sua relação com a saúde, tais como o desenvolvimento de câncer 10, aumento no apetite 11 e ganho de peso 12 , principalmente. Estudos nos quais a população-alvo é formada por indivíduos em geral são escassos; além disso, esses trabalhos têm seu foco na estimativa de ingestão diária de edulcorantes em decorrência do consumo de alimentos e bebidas diet 1,13 .

No Brasil, não foram encontrados estudos que forneçam estimativas confiáveis sobre a prevalência de utilização de adoçantes dietéticos pela população em geral, contudo algumas especulações sugerem que esteja ocorrendo um forte crescimento desses produtos no mercado brasileiro 14. Na Argentina, aproximadamente $14 \%$ da população utiliza regularmente adoçantes artificiais em bebidas e alimentos 15. Na Europa, o uso de adoçantes dietéticos parece estar aumentando, embora não tenha sido possível localizar estudos que informem a magnitude das prevalências. Esta tendência de aumento talvez possa ser explicada pelo crescente interesse em saúde e envelhecimento desta população ${ }^{8}$.

Em face da escassez de informações sobre o consumo de adoçantes dietéticos na população brasileira, faz-se necessário identificar a frequência de uso, assim como o perfil da população consumidora, independentemente de haver recomendação profissional ou não. O presente estudo tem o objetivo de avaliar o uso de adoçantes dietéticos pela população com idade igual ou superior a 20 anos, residentes na zona urbana do Município de Pelotas, Rio Grande do
Sul, Brasil. Este trabalho pretende trazer contribuições para a pesquisa em saúde e para a literatura brasileira, por meio de informações sobre a prevalência de utilização de adoçantes dietéticos na população em geral e sobre o perfil do usuário.

\section{Métodos}

No período de janeiro a julho de 2010, realizouse, em Pelotas, estudo observacional transversal de base populacional sobre a saúde de seus moradores. Pelotas está localizada na Região Sul do Brasil e conta com cerca de 340 mil habitantes. O presente trabalho é parte de um consórcio de pesquisa realizado entre os alunos do mestrado acadêmico do Programa de Pós-graduação em Epidemiologia da Universidade Federal de Pelotas (PPGE/UFPel), no qual todos os mestrandos desenvolvem seus estudos de maneira conjunta. Esta investigação incluiu indivíduos de ambos os sexos, com idades iguais ou superiores a 20 anos, moradores da zona urbana do Município de Pelotas. Indivíduos institucionalizados ou impossibilitados de responder ao questionário foram excluídos do estudo.

\section{Amostragem}

Com objetivo de otimizar a logística do trabalho de campo e, também, reduzir os custos deste processo, optou-se por utilizar uma amostra por conglomerados definidos de acordo com os setores censitários do Censo Demográfico 2000, fornecidos pelo Instituto Brasileiro de Geografia e Estatística (IBGE. http://www.ibge.gov.br).

O processo amostral foi realizado de modo a atender aos objetivos dos estudos de cada aluno do PPGE. Assim, verificou-se que o número necessário de domicílios a serem visitados seria 1.300. Na tentativa de reduzir os efeitos de delineamento, optou-se por amostrar, em média, 10 domicílios em cada um dos setores selecionados, totalizando 130 setores.

A amostragem foi realizada em dois estágios. Inicialmente, os 404 setores censitários fornecidos pelo IBGE foram listados em ordem crescente de renda média do chefe da família, e, com base nessa lista, foram sorteados 130 setores, com probabilidade proporcional ao tamanho, mediante uma amostragem sistemática. Em seguida, os domicílios pertencentes aos setores sorteados foram identificados e classificados de acordo com seu status de ocupação (residencial, comercial ou desocupado), sendo considerados elegíveis para fazer parte da amostra apenas os residenciais. Após esse processo, uma nova 
amostragem sistemática foi realizada para sortear os domicílios em cada conglomerado.

O cálculo de tamanho de amostra para o presente estudo foi realizado com base nos seguintes parâmetros: prevalência de uso de adoçantes dietéticos estimada em 50\% (uma vez que esta informação, em nível populacional, não foi encontrada na literatura); erro aceitável de três pontos percentuais; nível de $95 \%$ de confiança; efeito de delineamento de 2,0 e acréscimo de $10 \%$ para compensar possíveis perdas e recusas.

Dessa forma, a amostra final necessária foi de 2.346 indivíduos. Considerando-se que a estimativa de indivíduos na faixa etária de interesse $(\geq$ 20 anos) é de 2,14 por domicílio, seria necessário visitar 1.096 residências, todavia o total de domicílios visitados foi de 1.512, já que este trabalho está inserido em um consórcio de pesquisa.

\section{Definição do desfecho e variáveis} independentes

Considerou-se "usuário regular de adoçante dietético" aquele indivíduo que utilizou qualquer tipo de adoçante dietético em algum líquido, como café, chá, leite, iogurte ou suco, ingerido na semana anterior à entrevista, em pelo menos quatro dias. Este ponto de corte foi definido com base nos resultados de um estudo pré-piloto, o qual incluiu 30 indivíduos usuários de adoçantes. Do total de entrevistados, 93\% informaram ter utilizado o produto em pelo menos quatro dias da semana anterior.

Foram avaliadas as seguintes características: sexo, idade (anos completos), cor da pele (observada pela entrevistadora), escolaridade (anos completos de estudo), situação conjugal (solteiro/casado/separado/viúvo), nível econômico (definido conforme critério que leva em conta o poder de compra dos indivíduos - Associação Brasileira de Empresas de Pesquisa/2010 - dividido em cinco grupos, sendo o nível A o de maior poder aquisitivo) 16, presença de diabetes mellitus (sim/não), presença de hipertensão arterial sistêmica (sim/não) (ambas autorreferidas) e medidas antropométricas (peso e altura). A avaliação nutricional foi feita segundo critério da Organização Mundial da Saúde (OMS), que classifica o estado nutricional dos indivíduos de acordo com o índice de massa corporal - IMC $\left(\mathrm{kg} / \mathrm{m}^{2}\right)$, a saber: baixo peso (IMC $\left.<18,5\right)$, eutrofia (IMC $\geq 18,5$ e $\leq 24,9$ ), sobrepeso (IMC $\geq 25,0$ e $\leq$ 29,9) e obesidade (IMC $\geq 30,0) 17$.

\section{Instrumento de pesquisa}

O instrumento utilizado para avaliar o tema em estudo foi elaborado e pré-testado pelos próprios autores da pesquisa e abordou questões relacionadas ao uso de adoçantes dietéticos. Todas as opções de resposta eram fechadas (alternativas), exceto para a coleta das quantidades ingeridas. Para descrição do desfecho, foram realizadas as seis perguntas seguintes:

1) Desde < dia da semana $>$ da semana passada até hoje, o(a) Sr(a) usou adoçante artificial em algum líquido que bebeu?

2) Desde < dia da semana $>$ da semana passada até hoje, em quantos dias o(a) Sr(a) usou adoçante artificial nos líquidos que bebeu?

3) Qual a marca de adoçante que o(a) Sr(a) costuma usar nos líquidos, na maioria das vezes?

4) Em qual forma o(a) Sr(a) usa esse adoçante?

5) Que cor é a embalagem? (Para usuários de adoçante líquido).

6) Como é a embalagem? (Para usuários de adoçante em pó).

A partir das duas primeiras perguntas, pôdese dividir os indivíduos em usuários regulares (consumo $\geq 4$ dias na semana anterior à entrevista) e não usuários (consumo < 4 dias) de adoçantes. Em seguida, com base nas informações sobre a marca, forma de utilização e tipo de embalagem, foi possível avaliar os componentes (edulcorantes) dos adoçantes utilizados pelos usuários e, assim, classificá-los de acordo com o tipo (dietético ou não) consumido. Dessa forma, foram considerados positivos para o desfecho aqueles indivíduos que consumiram "adoçantes dietéticos" em "pelo menos quatro dias da última semana".

A estimativa de ingestão diária de adoçantes dietéticos foi obtida, da mesma forma, por meio da aplicação de seis perguntas:

1) Pense no último dia, que não seja hoje, que o(a) Sr(a) usou $<$ nome do adoçante $>$ nos líquidos. Durante todo esse dia, quantas vezes o(a) Sr(a) usou o $<$ nome do adoçante $>$ ?

2) Quantos(as) < forma de utilização> o(a) $\operatorname{Sr}($ a $)$ usou na la vez?

3) Quantos(as) < forma de utilização> o(a) $\operatorname{Sr}($ a $)$ usou na $2 \underline{a}$ vez?

4) Quantos(as) < forma de utilização> o(a) $\operatorname{Sr}(a)$ usou na $3 \underline{a}$ vez?

5) Quantos(as) < forma de utilização> o(a) $\operatorname{Sr}($ a $)$ usou na 4 a vez?

6) Quantos(as) <forma de utilização> o(a) $\operatorname{Sr}($ a $)$ usou na $5 \underline{a}$ vez?

As respostas obtidas para as cinco quantidades foram somadas; em seguida, calcularam-se as medianas de ingestão diária de gotas ou envelopes de adoçante, assim como seus respectivos intervalos interquartis; por fim, foram calculadas as medianas referentes à ingestão em apenas uma porção. 


\section{Coleta e análise dos dados}

Todos os dados foram coletados diretamente em um computador de bolso (Personal Digital Assistants - PDA) com capacidade para armazenamento e processamento de dados. No decorrer da pesquisa, foram realizadas checagens de amplitude e consistência, a fim de verificar possíveis erros de digitação. Para aferição das medidas antropométricas, foram utilizadas Balanças Digitais Solares HS301 (Tanita Corporation of America Inc., Arlington Heights, Estados Unidos), com capacidade máxima de $150 \mathrm{~kg}$ e precisão de $100 \mathrm{~g}$, e estadiômetros de alumínio de fabricação artesanal, com altura máxima de $2 \mathrm{~m}$ e precisão de $1 \mathrm{~mm}$. Esses estadiômetros foram idealizados pelo Centro de Pesquisas Epidemiológicas (PPGE/UFPel).

Todos os dados foram coletados por entrevistadoras previamente treinadas. Em adição, para a coleta das medidas, as antropometristas foram treinadas e submetidas a processo de padronização. A técnica utilizada para coleta de peso e altura seguiu as recomendações de Lohman et al. 18, e os erros técnicos aceitáveis das medidas foram baseados na publicação de Habitch 19 .

O banco de dados foi criado no programa Pendragon Forms 5.1 (Pendragon Software Corp., Buffalo Grove, Estados Unidos) para utilização direta no PDA, sendo dispensada a posterior digitação. As análises estatísticas foram realizadas com o auxílio do programa Stata versão 11.0 (Stata Corp., College Station, Estados Unidos). O desenho amostral foi definido de forma a corrigir as estimativas da variabilidade para correlação intraconglomerado. Foram realizados os testes qui-quadrado de heterogeneidade e de tendência linear para avaliar as diferenças entre os grupos.

O controle de qualidade do estudo foi realizado mediante revisita domiciliar a $10 \%$ dos indivíduos entrevistados, sorteados aleatoriamente. Para tanto, foi utilizado um questionário reduzido para verificação de possíveis erros ou respostas falsas; posteriormente, fez-se a avaliação da concordância e/ou repetibilidade das questões.

Este estudo foi aprovado pelo Comitê de Ética em Pesquisa da Faculdade de Medicina da Universidade Federal de Pelotas. Consentimento informado por escrito foi obtido dos participantes previamente à entrevista.

\section{Resultados}

Entre os 1.512 domicílios elegíveis para o estudo, foram identificados 3.059 indivíduos com idade igual ou superior a 20 anos, que atendiam aos cri- térios de inclusão para participação na pesquisa. Entretanto, a amostra final foi composta por 1.399 domicílios, totalizando 2.732 participantes. Um total de $10,7 \%$ da amostra (327 indivíduos) constituiu-se como não respondente (118 perdas e 209 recusas), sendo a maior parte do sexo masculino $(55,0 \%)$, cor da pele branca $(68,2 \%)$ e com idades entre 20 e 39 anos $(24,2 \%)$.

O controle de qualidade foi realizado com 299 indivíduos (10,9\% da amostra), para os quais se repetiu a pergunta sobre "utilização de adoçantes na última semana”. O resultado do teste kappa para avaliar a concordância e/ou repetibilidade da questão foi de 0,77 , considerado bom. O coeficiente de correlação intraclasse foi 0,03 , produzindo um efeito de delineamento amostral para uso de adoçantes dietéticos de 1,61.

A média de idade dos indivíduos da amostra foi de 46,1 anos (desvio-padrão - DP $\pm 17,0$ ), variando entre 20 e 96 anos. A Tabela 1 apresenta a descrição dos avaliados, bem como a prevalência de utilização de adoçantes dietéticos de acordo com características demográficas, socioeconômicas e de saúde. Observa-se que quase $58 \%$ dos entrevistados eram do sexo feminino, $81,2 \%$ tinham cor da pele branca e cerca de $59 \%$ declararam-se casados ou morando com companheiro. Mais de $46 \%$ da amostra referiu ter estudado pelo menos nove anos completos. Quanto ao nível econômico, dois terços da amostra pertenciam ao nível $\mathrm{C}$ ou inferior. No que se refere à condição de saúde, cerca de $10 \%$ dos indivíduos referiram ser diabéticos, e 36,7\% informaram ser hipertensos. Em relação ao estado nutricional, $62,4 \%$ dos indivíduos apresentaram excesso de peso (IMC $\geq 25,0 \mathrm{~kg} / \mathrm{m}^{2}$ ). Quando questionados sobre o uso geral de adoçantes na semana anterior à entrevista, 23,1\% (intervalo de 95\% de confiança - IC95\%: 21,0;25,2) dos indivíduos informaram ter utilizado o produto. Entre estes, a utilização de adoçante na semana anterior à entrevista variou entre $2,2 \%$ e $5,9 \%$ para o período entre 1-6 dias na semana, ao passo que $72,7 \%$ informaram consumir adoçantes diariamente. Assim, considerando-se a definição operacional do desfecho adotado no presente estudo, a prevalência de utilização de adoçante dietético diminuiu para 19,0\% (IC95\%: 17,1;20,9).

Observando-se ainda a Tabela 1, identificase que a prevalência de utilização de adoçante dietético foi mais frequente entre as mulheres $(22,7 \%)$ e indivíduos de cor da pele branca (19,7\%). Verifica-se, também, tendência de crescimento da prevalência de uso de adoçante dietético conforme o aumento da idade, sendo cerca de 3,7 vezes maior entre os idosos do que entre aqueles com idades entre 20 e 29 anos. Em relação à situação conjugal, observou-se associação 
Descrição da amostra e prevalência de utilização de adoçantes dietéticos entre os indivíduos avaliados, de acordo com características demográficas, socioeconômicas e de saúde. Pelotas, Rio Grande do Sul, Brasil, 2010 (N = 2.732 *).

\begin{tabular}{|c|c|c|c|c|c|}
\hline \multirow[t]{2}{*}{ Características } & \multicolumn{2}{|c|}{ Descrição da amostra } & \multicolumn{2}{|c|}{ Uso de adoçantes dietéticos } & \multirow{2}{*}{$\begin{array}{l}\text { Valor de p } \\
\qquad * \star \star\end{array}$} \\
\hline & $\mathbf{n}$ & $\%$ & Prevalência & $\mathrm{IC} 95 \%$ ** & \\
\hline Sexo & & & & & $<0,001$ \\
\hline Masculino & 1.151 & 42,1 & 13,9 & $11,9-15,9$ & \\
\hline Feminino & 1.581 & 57,9 & 22,7 & $20,2-25,3$ & \\
\hline Cor da pele & & & & & 0,05 \\
\hline Branca & 513 & 18,8 & 19,7 & $17,6-21,9$ & \\
\hline Não branca & 2.218 & 81,2 & 16,0 & $12,5-19,5$ & \\
\hline Idade (anos completos) & & & & & $<0,001 \#$ \\
\hline $20-29$ & 595 & 21,8 & 8,7 & $6,3-11,1$ & \\
\hline $30-39$ & 462 & 16,9 & 14,1 & $10,8-17,5$ & \\
\hline $40-49$ & 545 & 20,0 & 16,7 & $13,5-19,9$ & \\
\hline $50-59$ & 495 & 18,1 & 21,8 & $17,9-25,7$ & \\
\hline$\geq 60$ & 635 & 23,2 & 32,0 & $28,3-35,7$ & \\
\hline Situação conjugal & & & & & $<0,001$ \\
\hline Solteiro/Sem companheiro(a) & 644 & 23,6 & 14,3 & $16,7-21,3$ & \\
\hline Casado/Com companheiro(a) & 1.606 & 58,8 & 19,0 & $11,2-17,4$ & \\
\hline Separado(a) & 234 & 8,5 & 22,3 & $16,9-27,6$ & \\
\hline Viúvo(a) & 248 & 9,1 & 28,2 & $22,3-34,2$ & \\
\hline Escolaridade (anos de estudo) & & & & & $<0,001$ \\
\hline $0-4$ & 680 & 24,9 & 19,9 & $16,6-23,2$ & \\
\hline $5-8$ & 773 & 28,3 & 16,7 & $13,7-19,7$ & \\
\hline $9-11$ & 730 & 26,7 & 15,8 & $13,3-18,4$ & \\
\hline$\geq 12$ & 547 & 20,0 & 25,5 & $21,4-29,6$ & \\
\hline Nível econômico (ABEP) & & & & & $<0,001 \#$ \\
\hline$A / B$ & 931 & 34,1 & 28,9 & $24,5-33,3$ & \\
\hline C & 1.318 & 48,4 & 18,2 & $15,9-20,4$ & \\
\hline $\mathrm{D} / \mathrm{E}$ & 477 & 17,5 & 15,2 & $12,6-17,7$ & \\
\hline $\mathrm{IMC}\left(\mathrm{kg} / \mathrm{m}^{2}\right)$ & & & & & $<0,001 \#$ \\
\hline$<25,0$ & 922 & 37,6 & 11,2 & $9,1-13,3$ & \\
\hline $25,0-29,9$ & 888 & 36,3 & 20,6 & $17,6-23,6$ & \\
\hline$\geq 30,0$ & 638 & 26,1 & 28,6 & $24,6-32,5$ & \\
\hline Diabetes mellitus & & & & & $<0,001$ \\
\hline Não & 2436 & 89,9 & 15,2 & $13,4-17,1$ & \\
\hline Sim & 273 & 10,1 & 54,2 & $48,1-60,3$ & \\
\hline Hipertensão arterial sistêmica & & & & & $<0,001$ \\
\hline Não & 1.716 & 63,3 & 13,7 & $11,9-15,5$ & \\
\hline Sim & 993 & 36,7 & 28,6 & $25,3-31,9$ & \\
\hline
\end{tabular}

ABEP: Associação Brasileira de Empresas de Pesquisa.

* O valor máximo de informações perdidas foi de 10,4\% (284) para a variável IMC (índice de massa corporal);

** Intervalo de 95\% de confiança (IC95\%) ajustado para efeito de delineamento amostral;

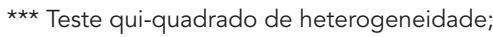

\# Teste qui-quadrado de tendência linear.

estatisticamente significativa, com maior prevalência de uso entre indivíduos viúvos quando comparados aos solteiros ou sem companheiro. No que se refere à escolaridade, verificou-se consumo aproximadamente $30 \%$ mais elevado entre indivíduos com 12 anos ou mais de estudo do que entre aqueles na categoria de mais baixa escolaridade. As variáveis nível econômico e 
estado nutricional apresentaram associação direta e significativa com a utilização de adoçantes dietéticos. Ademais, indivíduos diabéticos e hipertensos apresentaram prevalências de utilização de adoçante dietético aproximadamente 3,5 e 2,0 vezes maior, respectivamente, quando comparados àqueles sem a doença.

A Tabela 2 descreve as características relacionadas ao adoçante dietético utilizado e mostra que quase $98 \%$ dos usuários relataram seu uso na forma líquida, sendo os mais consumidos $(89,2 \%)$ aqueles compostos pelos edulcorantes artificiais - sacarina sódica e ciclamato de sódio. Apenas 3,3\% dos usuários referiram utilizar adoçante dietético à base de edulcorante natural (esteviosídeo). Quando questionados sobre quem recomendou a utilização de adoçante dietético, 54,3\% dos usuários referiram não ter recebido recomendação de ninguém. Entre aqueles que receberam recomendação, o médico foi o mais citado (76,4\%).

A Tabela 3 apresenta a distribuição das características sociodemográficas e de saúde entre os usuários regulares de adoçantes dietéticos. Podese observar que este grupo foi composto predo- minantemente por indivíduos do sexo feminino, cor da pele branca e idade igual ou superior a 60 anos. Além disso, quase $60 \%$ dos usuários regulares de adoçantes dietéticos declararam que eram casados ou que moravam com companheiro. Ainda nesse contexto, a Figura 1 apresenta a distribuição das morbidades avaliadas entre os usuários regulares de adoçantes dietéticos. Notase que as morbidades mais frequentes foram excesso de peso e hipertensão arterial sistêmica de forma conjunta $(28,7 \%)$, seguidas pelo excesso de peso isoladamente $(26,1 \%)$ e pela tríade diabetes mellitus, hipertensão arterial sistêmica e excesso de peso (18,2\%). Cabe destacar que aproximadamente $14 \%$ dos usuários regulares de adoçantes dietéticos não apresentavam nenhuma das morbidades avaliadas no momento da entrevista.

Em relação à quantidade de adoçante dietético utilizada, observou-se que a mediana de ingestão diária foi de dez gotas (P25; P75 = 6; 18); para os usuários de adoçante em pó, a mediana foi de 1,5 sachet $(\mathrm{P} 25 ; \mathrm{P} 75=1 ; 4)$. No que se refere à quantidade consumida em apenas uma porção, a mediana de utilização foi de seis gotas (P25; P75 = 4; 10); para usuários de adoçante em pó, a

\begin{tabular}{|c|c|c|}
\hline Característica & n & $\%$ \\
\hline \multicolumn{3}{|l|}{ Forma de uso } \\
\hline Líquida & 507 & 97,7 \\
\hline Pó & 12 & 2,3 \\
\hline \multicolumn{3}{|l|}{ Composição } \\
\hline Sacarina sódica + ciclamato de sódio & 463 & 89,2 \\
\hline Multiadoçante dietético * & 18 & 3,4 \\
\hline Esteviosídeo & 17 & 3,3 \\
\hline Aspartame & 21 & 4,1 \\
\hline \multicolumn{3}{|l|}{ Recomendação para uso } \\
\hline Não & 282 & 54,3 \\
\hline $\operatorname{Sim}$ & 237 & 45,7 \\
\hline \multicolumn{3}{|l|}{ Quem recomendou? $(\mathrm{n}=237)$} \\
\hline Médico & 181 & 76,4 \\
\hline Nutricionista & 47 & 19,8 \\
\hline Familiar & 9 & 3,8 \\
\hline \multicolumn{3}{|l|}{ Utilização na forma de esguicho $(n=503)$ ** } \\
\hline Não & 451 & 89,7 \\
\hline Sim & 52 & 10,3 \\
\hline
\end{tabular}

* Adoçantes compostos por três tipos de edulcorantes pelo menos: sacarina sódica, ciclamato de sódio, aspartame ou acessulfame de potássio;

** Informação perdida para quatro indivíduos. 
Descrição das características estudadas entre os usuários regulares de adoçantes dietéticos. Pelotas, Rio Grande do Sul, Brasil, $2010(n=519)$.

\begin{tabular}{|c|c|c|}
\hline Característica & $\mathrm{n}$ & $\%$ \\
\hline \multicolumn{3}{|l|}{ Sexo } \\
\hline Masculino & 160 & 30,8 \\
\hline Feminino & 359 & 69,2 \\
\hline \multicolumn{3}{|l|}{ Cor da pele } \\
\hline Não branca & 82 & 15,8 \\
\hline Branca & 437 & 84,2 \\
\hline \multicolumn{3}{|l|}{ Idade (anos completos) } \\
\hline $20-29$ & 52 & 10,0 \\
\hline $30-39$ & 65 & 12,5 \\
\hline $40-49$ & 91 & 17,6 \\
\hline $50-59$ & 108 & 20,8 \\
\hline$\geq 60$ & 203 & 39,1 \\
\hline \multicolumn{3}{|l|}{ Situação conjugal } \\
\hline Casado(a)/Com companheiro(a) & 305 & 58,8 \\
\hline Solteiro(a)/Sem companheiro(a) & 92 & 17,7 \\
\hline Separado(a) & 52 & 10,0 \\
\hline Viúvo(a) & 70 & 13,5 \\
\hline \multicolumn{3}{|l|}{ Escolaridade (anos de estudo) } \\
\hline $0-4$ & 135 & 26,1 \\
\hline $5-8$ & 129 & 24,9 \\
\hline $9-11$ & 115 & 22,2 \\
\hline$\geq 12$ & 139 & 26,8 \\
\hline \multicolumn{3}{|l|}{ Nível econômico (ABEP) } \\
\hline$A+B$ & 138 & 26,6 \\
\hline C & 239 & 46,2 \\
\hline$D+E$ & 141 & 27,2 \\
\hline
\end{tabular}

ABEP: Associação Brasileira de Empresas de Pesquisa.

mediana manteve-se em 1,5 sachet $(\mathrm{P} 25 ; \mathrm{P} 75=1$; 2). Alguns (10\%) usuários de adoçante dietético líquido referiram sua utilização na forma de "esguicho”, em vez de informar o número de gotas. Para estes, não foi possível quantificar a ingestão de adoçante; portanto, suas informações foram desconsideradas nos cálculos de estimativa de ingestão.

\section{Discussão}

Estudos de base populacional sobre o uso de adoçantes dietéticos são escassos na literatura científica. A maioria das pesquisas relacionadas a adoçantes busca estimar a ingestão diária de edulcorantes com base no consumo de produtos diet/light 1,13, ou avaliar a associação entre seu consumo a longo prazo e problemas de saúde, tais como desenvolvimento de câncer 10, aumento do apetite 11 e ganho de peso ${ }^{12}$. Além disso, tais pesquisas costumam ser realizadas em grupos específicos de populações 13,20. Este parece ser o primeiro estudo de base populacional realizado no país com o objetivo de investigar a prevalência de utilização de adoçantes dietéticos entre adultos.

O acelerado aumento das doenças crônicas não transmissíveis, como a obesidade, ocorrido nas últimas décadas pode estar relacionado a estilos de vida não saudáveis, incluindo práticas alimentares inadequadas (consumo excessivo de açúcares e gorduras) e sedentarismo 21 . Indivíduos que necessitam perder ou controlar seu peso apresentam indicação para uso de adoçantes dietéticos em substituição ao açúcar como uma ferramenta auxiliar em um programa de reeducação alimentar 8 . 
Distribuição das morbidades avaliadas entre os usuários regulares de adoçantes dietéticos. Pelotas, Rio Grande do Sul, Brasil $\left(n=467^{\star}\right)$.

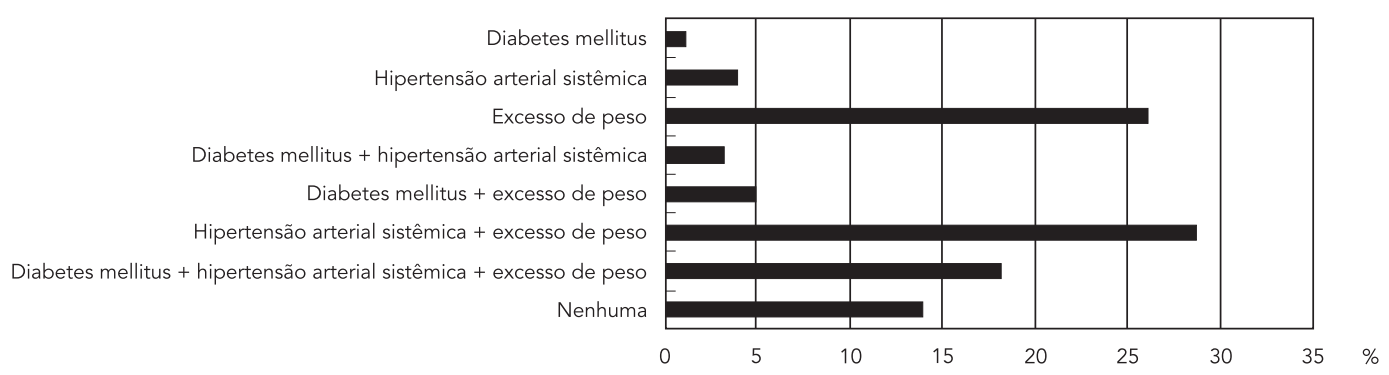

* Incluídos apenas os usuários de adoçantes dietéticos para os quais se dispunha de informações sobre as três morbidades avaliadas.

A magnitude da prevalência (19\%, IC95\%: $17,1 ; 20,9)$ de uso de adoçante dietético verificada no presente estudo é semelhante àquelas encontradas na literatura em populações de outros países. Dados da Secretaría de Agricultura, Ganadería, Pesca y Alimentación (SAGPyA) da Argentina mostram que $14 \%$ da população usa regularmente adoçantes artificiais para adoçar seus alimentos e bebidas 15. Nos Estados Unidos, a pesquisa National Health and Nutrition Examination Survey (NHANES), referente ao consumo de alimentos e bebidas que contêm edulcorantes não calóricos, demonstrou que $15 \%$ da população referiu ter consumido algum alimento ou bebida contendo edulcorantes não calóricos no período entre 2003 e 2004 6. Na França, estudo de coorte realizado com adultos de 45-60 anos de idade encontrou prevalência de $17 \%$ de uso de adoçante artificial por essa população. Neste estudo, a avaliação do uso de adoçante dietético foi realizada com base na aplicação de seis inquéritos do tipo recordatório de 24 horas ao longo de um ano (um inquérito a cada dois meses), sendo considerados usuários regulares os indivíduos que referiram a utilização desses produtos em, pelo menos, quatro dos seis inquéritos realizados 22. É importante destacar que essas comparações devem ser realizadas com cautela, tendo em vista suas diferenças metodológicas quanto aos instrumentos utilizados, população em estudo e período recordatório de cada pesquisa.

A prevalência de utilização de adoçantes dietéticos mostrou-se associada a variáveis demográficas, socioeconômicas e de saúde. No presente estudo, o uso de adoçantes dietéticos foi significativamente maior entre as mulheres quando comparadas aos homens. O supracitado estudo francês mostrou que aproximadamente $20 \%$ das mulheres utilizaram adoçante dietético em, pelo menos, quatro dos seis inquéritos aplicados durante o ano 22 . Atualmente, existe um forte estímulo ao desenvolvimento e à manutenção de estilos de vida saudáveis, em que o controle de peso está inserido. Some-se a isso o fato de que as mulheres sentem-se cada vez mais pressionadas a fazer dieta e acabam por submeterem-se a restrições alimentares rígidas, a fim de atingir um padrão estético socialmente imposto. O consumo de produtos dietéticos está associado a tais práticas para redução de peso 23 .

Em relação à idade, o uso de adoçante dietético foi quase quatro vezes maior entre os idosos, quando comparados aos mais jovens. Estudo realizado com indivíduos diabéticos em Ribeirão Preto, São Paulo, Brasil, encontrou que a frequência de uso de adoçantes dietéticos também foi maior entre idosos ( $\geq 65$ anos), do que entre os indivíduos com idades entre 30 e 64 anos 24 . A idade é uma característica que pode exercer influência sobre o uso de adoçantes dietéticos de maneira diferente, conforme o grupo etário. Indivíduos mais jovens, geralmente, utilizam adoçantes dietéticos para manutenção do peso ou simplesmente para acompanhar o hábito de familiares 1,11. À medida que a idade aumenta, a motivação para utilização de adoçantes dietéticos muda, sendo, então, a perda de peso e a presença de doenças as principais razões 25 .

O nível econômico e a escolaridade são características relacionadas entre si e ambas podem influenciar o uso de adoçantes dietéticos de forma semelhante. Observou-se, no presente 
estudo, que indivíduos pertencentes aos níveis econômicos mais altos ou com maior escolaridade apresentaram maiores prevalências de uso de adoçante dietético. Talvez essa relação possa ser explicada pela maior conscientização que esses indivíduos têm sobre a importância da manutenção de boa saúde, tendo em vista que alimentos diet e light, assim como adoçantes dietéticos, são vistos por algumas pessoas como produtos saudáveis 25 . De forma geral, o comportamento alimentar desses indivíduos em relação ao consumo desse tipo de produto pode ser explicado pela preocupação com a forma física ou estética, mais frequente em níveis sociais mais elevados 23 .

Neste trabalho, também foi avaliada a relação entre o uso de adoçantes dietéticos e a presença de algumas morbidades específicas. Os resultados mostraram que a prevalência de utilização de adoçante dietético foi maior entre diabéticos, hipertensos ou indivíduos com excesso de peso. Estudo realizado por Bellisle et al. 22 mostrou que o consumo de produtos reduzidos em açúcar foi maior entre indivíduos que apresentavam maior IMC. Castro \& Franco 20 verificaram que, entre diabéticos, a prevalência de uso de adoçante dietético foi cerca de $90 \%$; todavia, antes do diagnóstico da doença, apenas $6 \%$ desses indivíduos faziam uso de tais produtos. Por ser um estudo transversal, esses resultados poderiam estar sujeitos ao viés de causalidade reversa. Dessa forma, nenhuma associação foi interpretada como se tivesse uma relação causal. A utilização de adoçante dietético nesses casos pode estar sendo coadjuvante no tratamento dessas doenças ou estar sendo usado de forma preventiva para outras complicações, podendo seu uso ter sido recomendado ou não por algum profissional de saúde 5,11,20. É interessante destacar que, no presente estudo, $14 \%$ dos usuários regulares de adoçante dietético parecem não usar tais produtos para fins terapêuticos, pois não apresentam nenhuma das morbidades investigadas. Novos estudos, especialmente com delineamento qualitativo, poderiam esclarecer a motivação desses indivíduos que optam pelo uso de adoçantes dietéticos.

No que se refere às características relacionadas ao tipo de adoçante utilizado, cerca de $98 \%$ dos usuários relataram consumi-lo na forma líquida. Os motivos para utilização de adoçante dietético com tal apresentação podem estar relacionados com a praticidade e facilidade de uso que a forma líquida proporciona 20. Quanto à sua composição, aproximadamente $90 \%$ dos usuários referiram o uso de adoçantes à base de sacarina sódica e ciclamato de sódio, possivelmente por serem os edulcorantes mais antigos, apresentarem menor custo e serem os mais divulgados pela mídia 20 . Cabe destacar que o edulcorante ciclamato de sódio foi banido dos Estados Unidos em 1970 e tem sua utilização proibida até hoje 26 . Apesar das divergências encontradas sobre a segurança sanitária dos edulcorantes da "primeira geração" (sacarina, ciclamato e aspartame), a maioria dos estudos que investigou a segurança desses produtos para o consumo humano não encontrou evidência que sustente a hipótese de que apresentem risco à saúde 27,28. Por sua vez, os adoçantes mais novos, compostos pelos edulcorantes da "segunda geração" (acessulfame de potássio, esteviosídeo, sucralose, neotame e taumatina), não apresentam qualquer evidência contrária ao seu consumo ${ }^{29}$. Além disso, a sucralose teve sua segurança sanitária avaliada em estudo recente, e seu uso entre indivíduos diabéticos, crianças e gestantes foi considerado seguro 30 .

Grande parte dos usuários de adoçante dietético $(54,3 \%)$ referiu consumir tais produtos sem ter havido qualquer recomendação por um profissional de saúde. A ANVISA, com base em recomendação da OMS, desaconselha o uso de adoçantes à base de sacarina e ciclamato para pacientes hipertensos, em virtude da elevada concentração de sódio desses edulcorantes 31 . Para pacientes diabéticos e/ou hipertensos, a literatura 32 sugere que os mais recomendados sejam os adoçantes à base de stévia, tendo em vista suas propriedades terapêuticas naturais como anti-hipertensivo e anti-hiperglicemiante. Outra recomendação importante é que indivíduos fenilcetonúricos não devem utilizar adoçantes à base de aspartame, por causa da presença do aminoácido fenilalanina 4,5. Em adição, o aspartame fornece $4 \mathrm{kcal} / \mathrm{g}$ (tanto quanto a sacarose) e, por isso, deveria ser consumido em pequenas quantidades 26 .

A utilização de adoçante na forma de "esguichos" em vez de gotas foi referida por cerca de $10 \%$ dos usuários de adoçante dietético líquido. No estudo realizado por Castro \& Franco 21, incluindo apenas pacientes diabéticos, 16,1\% desses usuários referiram utilizá-lo na forma de "esguicho", sugerindo que esses indivíduos não estão preocupados com a quantidade ingerida, ou não foram adequadamente orientados sobre a correta utilização do produto. O estudo desses autores foi o único encontrado na literatura que menciona o uso de adoçantes na forma de “esguicho”, no entanto a comparação com o presente estudo deve ser cautelosa por se tratar de populações diferentes.

Avaliar a ingestão diária de edulcorantes é uma tarefa difícil, pois essas substâncias podem ser encontradas nos mais diversos tipos de alimentos, bebidas, e até mesmo na composição 
química de medicamentos ${ }^{8}$. Sabe-se que o consumo de produtos dietéticos, principalmente refrigerantes diet e adoçantes dietéticos, são as principais fontes de ingestão de edulcorantes 1 . Assim, a comparação entre a ingestão diária dessas substâncias e a ingestão diária aceitável (IDA), preconizada pelo Ministério da Saúde, é uma limitação do presente estudo, tendo em vista que o instrumento utilizado na pesquisa não contemplou o consumo de outros alimentos que contêm edulcorantes. Ademais, as informações sobre a ingestão de adoçantes foram coletadas apenas para um dia, o que reduz a precisão dessa estimativa, pois pode haver oscilação nas quantidades de acordo com os dias da semana, tipo e quantidade do líquido ingerido. Finalmente, a maioria dos adoçantes dietéticos possui pelo menos dois edulcorantes em sua composição, não sendo possível avaliar a ingestão de cada um separadamente.

O presente estudo mostrou que a utilização de adoçante dietético foi maior, principalmente, entre as mulheres, indivíduos idosos e de maior nível econômico, assim como para aqueles de maior escolaridade e com excesso de peso. Com base nos resultados apresentados, recomenda-se que os usuários busquem orientação profissional para que os adoçantes sejam utilizados de maneira adequada e segura para a saúde. É importante que os profissionais de saúde tenham conhecimento suficiente para orientar de forma adequada quanto ao correto modo de uso e ao melhor tipo de adoçante para a necessidade específica de cada paciente.

\section{Resumo}

Estudo de base populacional avaliou o uso de adoçantes dietéticos na população com idade $\geq 20$ anos, residente na zona urbana do Município de Pelotas, Rio Grande do Sul, Brasil. A coleta de dados ocorreu entre janeiro e julho de 2010, e 2.732 indivíduos foram entrevistados. Além das questões específicas quanto ao uso de adoçante dietético, foram coletadas informações sobre características demográficas, socioeconômicas e de saúde. Para as análises estatísticas, foram utilizados testes qui-quadrado de heterogeneidade e de tendência linear. A prevalência de uso de adoçante dietético foi 19\% (IC95\%: 17,1;20,9), sendo 3,7 vezes maior entre idosos do que entre aqueles com 20-29 anos de idade. Nível econômico e estado nutricional apresentaram associação direta e significativa com o desfecho. Quase 98\% da amostra utilizou adoçantes na forma líquida, sendo os mais consumidos $(89,2 \%)$ aqueles constituídos por sacarina e ciclamato de sódio. A mediana de ingestão diária foi 10 gotas (P25; P75 $=6 ; 18$ ), entre usuários de adoçante líquido, ou 1,5 sachet (P25; P75 = 1; 4), para adoçante em pó. O uso de adoçante dietético foi maior entre mulheres e idosos.

Adoçantes Dietéticos; Edulcorantes; Prevalência

\section{Colaboradores}

R. V. Zanini participou de todas as etapas do trabalho de campo e foi responsável pela revisão da literatura, análise dos dados e redação do manuscrito. C. L. Araújo orientou todas as etapas da pesquisa e revisou o manuscrito. J. Martínez-Mesa colaborou na revisão e análise dos dados.

\section{Agradecimentos}

Nossos agradecimentos à Coordenação de Aperfeiçoamento de Pessoal de Nível Superior (CAPES) pelo apoio financeiro. 


\section{Referências}

1. Toledo MC, Ioshi SH. Potential intake of intense sweeteners in Brazil. Food Addit Contam 1995; 12:799-808.

2. Ministério da Saúde. Portaria $n^{\circ} .25$, de 04 de abril de 1988. Os produtos à base de edulcorantes, com ou sem adição de açúcar, passam a denominarse “Adoçantes Dietéticos”. Diário Oficial da União 1988; 4 jun.

3. Ministério da Saúde. Portaria no. 38, de 13 de janeiro de 1998. Aprova o regulamento técnico referente a adoçantes de mesa, constante do anexo desta portaria. Diário Oficial da União 1998; 2 abr.

4. Ministério da Saúde. Portaria no. 29, de 13 de janeiro de 1998. Aprova o regulamento técnico referente a alimentos para fins especiais. Diário Oficial da União 1998; 15 jan.

5. Rosado EL, Monteiro JBR. Obesidade e a substituição de macronutrientes da dieta. Rev Nutr 2001; 14:145-52.

6. Mattes RD, Popkin BM. Nonnutritive sweetener consumption in humans: effects on appetite and food intake and their putative mechanisms. Am J Clin Nutr 2009; 89:1-14.

7. Popkin BM, Nielsen SJ. The sweetening of the world's diet. Obesity 2003; 11:1325-32.

8. American Dietetic Association. Position of the American Dietetic Association: use of nutritive and nonnutritive sweeteners. J Am Diet Assoc 2004; 104:255-75.

9. Agência Nacional de Vigilância Sanitária. Resolução RDC nº. 18, de 24 de março de 2008. Regulamento Técnico que autoriza o uso de aditivos edulcorantes em alimentos, com seus respectivos limites máximos. Diário Oficial da União 2008; 25 mar.

10. Bosetti C, Gallus S, Talamini R, Montella M, Franceschi S, Negri E, et al. Artificial sweeteners and the risk of gastric, pancreatic, and endometrial cancers in Italy. Cancer Epidemiol Biomarkers Prev 2009; 18:2235-8.

11. Benton D. Can artificial sweeteners help control body weight and prevent obesity? Nutr Res Rev 2005; 18:63-76.

12. Swithers SE, Martin AA, Davidson TL. High-intensity sweeteners and energy balance. Physiol Behav 2010; 100:55-62.

13. Garnier-Sagne I, Leblanc JC, Verger P. Calculation of the intake of three intense sweeteners in young insulin-dependent diabetics. Food Chem Toxicol 2001; 39:745-9.

14. Associação Brasileira da Indústria de Alimentos Dietéticos e Para Fins Especiais. Mercado diet e light novo, 2004. http://www.abiad.org.br/pdf/ mercado_diet_light_novo.pdf (acessado em 29/ Mai/2009).

15. Secretaría de Agricultura, Ganadería, Pesca y Alimentación. Análisis de la cadena de edulcorantes. Dirección de Industria Alimentaria; 2000. http://www.alimentosargentinos.gov.ar/0-3/azu car/edulco/Edulcorantes.htm (acessado em 21/ Ago/2009).

16. Associação Brasileira de Empresas de Pesquisa. Critério brasileiro de classificação econômica. São Paulo: Associação Brasileira de Empresas de Pesquisa; 2010.
17. World Health Organization. Physical status: the use and interpretation of anthropometry. Report of a WHO Expert Committee. Geneva: World Health Organization; 1995. (Technical Report Series, 854).

18. Lohman TG, Roche AF, Martrell R. Anthropometric standardization reference manual. Champaign: Human Kinetics; 1988.

19. Habicht JP. Estandarización de métodos epidemiológicos cuantitativos sobre el terreno. Bol Oficina Sanit Panam 1974; 76:375-84.

20. Castro AGP, Franco LJ. Caracterização do consumo de adoçantes alternativos e produtos dietéticos por indivíduos diabéticos. Arq Bras Endocrinol Metab 2002; 46:280-7.

21. Joint WHO/FAO Expert Consultation. Diet, nutrition and the prevention of chronic diseases. Geneva: World Health Organizatin; 2003. (WHO Technical Report Series, 916).

22. Bellisle F, Altenburg-de-Assis MA, Fieux B, Preziosi P, Galan P, Guy-Grand B, et al. Use of "light” foods and drinks in French adults: biological, anthropometric and nutritional correlates. J Hum Nutr Diet $2001 ; 14: 191$.

23. Lucchese T, Batalha MO, Lambert JL. Marketing de alimentos e o comportamento de consumo: proposição de uma tipologia do consumidor de produtos light e/ou diet. Organizações Rurais e Agroindustriais 2006; 8:227-39.

24. Oliveira PB, Franco LJ. Consumo de adoçantes e produtos dietéticos por indivíduos com diabetes melito tipo 2, atendidos pelo Sistema Único de Saúde em Ribeirão Preto, SP. Arq Bras Endocrinol Metab 2010; 54:455-62.

25. Viaene J, Gellynck X. Consumer behaviour towards light products in Belgium. British Food Journal 1997; 99:105-13.

26. Manfred K, Kathleen M, Ruth K. Low-calorie sweeteners and other sugar substitutes: a review of the safety issues. Compr Rev Food Sci Food Saf 2006; 5:35-47.

27. Kenji W, Yoshiyuki O, Kohji O, Kunio A. Prognostic significance of selected lifestyle factors in urinary bladder cancer. Jpn J Cancer Res 1993; 84:1223-9.

28. Weihrauch MR, Diehl V. Artificial sweeteners: do they bear a carcinogenic risk? Ann Oncol 2004; 15:1460-5.

29. Gallus S, Scotti L, Negri E, Talamini R, Franceschi S, Montella M, et al. Artificial sweeteners and cancer risk in a network of case-control studies. Ann Oncol 2007; 18:40-4.

30. Grotz VL, Munro IC. An overview of the safety of sucralose. Regul Toxicol Pharmacol 2009; 55:1-5.

31. Instituto Nacional de Metrologia, Normalização e Qualidade Industrial. Adoçantes de mesa e adoçantes dietéticos; 2006. http://www.inmetro.gov. br/consumidor/produtos/adocantes.asp\#inf (acessado em 25/Set/2010).

32. Chatsudthipong V, Muanprasat C. Stevioside and related compounds: therapeutic benefits beyond sweetness. Pharmacol Ther 2009; 121:41-54.

Recebido em 12/Nov/2010

Versão final reapresentada em 21/Mar/2011

Aprovado em 29/Mar/2011 